\title{
Correction to: Education, Democracy and Inequality
}

Correction to:

B. Hoskins, J. G. Janmaat, Education, Democracy and Inequality, https:// doi.org/10.1057/978-1-137-48976-0

The following sentence has been included in the Acknowledgment section.

"The research for this book forms part of the research agenda of the Centre for Learning and Life Chances (LLAKES), which has been funded by the Economic and Social Research Council since 2008 - grant reference number: ES/J019135/1. We are grateful for the support of LLAKES, which proved vital for the completion of the book."

The updated version of the book can be found at https://doi.org/10.1057/978-1-137-48976-0 